\title{
Designing physical topologies for wavelength-routed all-optical networks
}

\author{
D. R. Hjelme $e^{1,2}$ and S. Eikedal Andersen ${ }^{l}$ \\ ") Department of Physical Electronics, Faculty of Electrical Engineering and \\ Telecommunications, Norwegian University of Science and Technology \\ ${ }^{2)}$ Department of Photonics, SINTEF Electronics and Cybernetics \\ N-7034 Trondheim, Norway \\ Tel: +4773592722, Fax: +4773591441 \\ E-mail:Dag.R.Hjelme@ecy.sintef.no
}

\begin{abstract}
The influence of the physical topology design on the overall network cost is studied. Both optical cross connect cost and link cost, including cable and fiber/amplifiers, are considered. A number of regular and non-regular topologies with 9 to 24 nodes and node degrees between 2 and 6 are studies by computing the average number of hops, required to meet a uniform traffic demand, as function of node degrees and number of nodes. By relating the average number of hops to the most important cost factors, it is shown how the best topology (lowest cost) shifts from a sparsely connected topology if the cable cost dominates, to medium connected topology if the fiber cost is dominating, to densely connected topologies if the node cost is dominating. The lowest average number of hops, close to that of the ideal spanning tree, are found for highly non-regular topologies requiring long link lengths. If mapped on to a regular grid and using fiber by-passes at the node location such optimized topologies can be used to reduce the node complexity.
\end{abstract}

Keywords

Optical network, physical topology, wavelength division multiplexing

1 INTRODUCTION

Optimization and evaluation of the physical topology for wavelength routed optical networks (WRONs) is a difficult problem that is usually treated numerically. However, many of the network characteristics are to a large extent determined by a few average network parameters. For example, we have earlier shown (Hjelme 1996) that much insight can be gained using simple approximate analytical corrected. The Erratum to this chapter is available at DOI: 10.1007/978-0-387-35398-2_19 


\section{Part Seven: Advanced Topics in optical networks}

results relating a set of average network parameters. On reason that such simple analytical results work well, is that the design problem has a very large number of degrees of freedom. Often one finds that many apparently different designs performs very similarly. By reducing the number of degrees of freedom by limiting one self to global (or average) network parameters, we are able to focus on the dominating factors without being blinded by details.

Both the number of wavelengths and the number of hops required to satisfy the traffic demand are factors determining both feasibility and cost of the network design. The maximum values can be used to evaluate the feasibility, whereas the average values can be used to estimate the overall network implementation cost.

It has been shown that the physical connectivity of a network determines the wavelength requirements (Hjelme 1996, Baroni 1997); more densely connected networks require fewer wavelengths. In this paper we will focus on the average number of hops required, and show that this parameter determines many of the more important network characteristics. We will demonstrate that by knowing this parameters combined with a few simple analytical relationships, information on both transmission system requirements, node complexity, and network cost can be obtained.

\section{SIGNIFICANCE OF THE AVERAGE NETWORK PARAMETERS}

To be able to compare various topologies as candidates for WRONs, we have to consider both link dimensions, node complexity, and network cost.

\subsection{Link dimensions}

We consider a network with $N$ nodes and $N_{L}$ links. Each link consists of $F_{i}\left(i=1\right.$ to $\left.N_{L}\right)$ fibers with $W_{i j}$ ( $i=1$ to $N_{L}$ and $j=1$ to $F_{i}$ ) wavelengths. If we sum over all link capacities, we can express the total number of wavelength channels in the network as $1 / 2 N D F W$, where $D$ is the average node degree, $F$ is the average number of fibers per link, and $W$ is the average number of wavelengths per fiber. These wavelength channels are used to route the traffic demand $T_{i}(i=1$ to $N(N-1) / 2)$, in terms of wavelength channels, each requiring $H_{i}$ hops, on all $N(N-1) / 2$ bi-directional connections in the network. We define hops to be equal to the number of links traversed for one connection. If we sum over all connections, we can express the total number of wavelength channels needed to route the traffic as $1 / 2 N(N-1) T H$, where $T H$ is the average number of channels times the number of hops per connection.

The number of wavelength channels available in the network must be equal or larger than the number of channels required to route the traffic demand such that we can write the inequality

$$
W D F /(N-1) T \geq H \text {. }
$$

We can use the equal sign only if there is no spare capacity. The amount of spare capacity required depends on the protection and restoration strategies used, a topic that is beyond the scope of this paper. 
We have earlier shown that for a large class of topologies the ratio between the network diameter and the average link length, $L$, is given by (Hjelme 1996)

network diameter $/ L \propto H$

Our first conclusion is therefore that designing topologies that minimizes the average number of hops will reduce the transmission system requirement (with respect to number of wavelengths and fibers). We note that such designs will generally require longer link lengths than other topologies.

\subsection{Node complexity}

The left hand side of (1) is identical to the ratio between the total average node throughput traffic and the local add/drop traffic. Therefore, we can write

throughput traffic / local add-drop traffic $\geq H$

Furthermore, using relationship (1), we can show (Hjelme 1997, Limal 1998) that the number of components in the optical cross connect (OXC) scales with $H$ to the power one or two (depending on which components and OXC architecture (Jordan 1996) are considered), such that we can write

OXC component count $\propto H^{\gamma}, \quad \gamma=1$ or 2

Our second conclusion is therefore that designing topologies that minimizes the average number of hops will i) reduce the required node throughput and ii) reduce the node complexity ( $\propto$ number of components required in the $\mathrm{OXC}$ ).

\subsection{Network cost}

We consider three contributions to the overall network cost i) fiber cost, ii) cable cost, and iii) OXC cost. The total link length scales with $D L$, and the total fiber length scales with $W D L \propto H L$. The OXC cost scales with the component number count, i.e. with $H^{\gamma}$. It then follows that if the overall cost of the network is dominated by

- the cost per fiber in the link, the overall network cost scales with $H L$,

- the cost per link (cable), the overall cost scales with $D L$, and

- the OXC cost the overall cost scales with $H^{\gamma}$.

The best topology for a given set of nodes and traffic demand therefore depends on the relative importance of these three factors. 
Our third conclusion is therefore that that designing topologies that minimizes the average number of hops will help minimize the overall network cost.

\section{THE RELATIONSHIP BETWEEN PHYSICAL TOPOLOGY AND AVERAGE NUMBER OF HOPS REQUIRED}

$H$ is determined by both the topology and the routing strategy used. In most cases, one will use a shortest path routing strategy. The choice of topology on the other hand is not obvious. The design space range from basic ring structures to fully meshed structures. For meshed topologies the choices include both node degree, i.e. the number of links to use, as well as the interconnect pattern, i.e. how we use the links to interconnect the nodes.

To learn more about the influence of the physical topology on the overall network cost we have computed the average number of hops required to route a uniform traffic demand (all-to-all connections) for a number of different physical topologies. The average number of hops was computed using Dijkstra's shortest path (in terms of hops) algorithm. We considered networks with number of nodes ranging from 9 to 24 with average node degrees ranging from 2 to 6 . Both regular topologies and topologies designed to reduce the average number of hops were considered (minimum- $H$ topologies). Our objective was to find out what can be gained by using the more complex minimum- $H$ topologies compared to regular topologies.

The minimum- $H$ topologies used here were designed with the objective to have an ideal spanning three from each node, i.e. a topology where the source node is one hop away from $D$ nodes, which is one hop away from $D-1$ nodes and so on (Hluchyj 1991). We used an iterative procedure to design the topologies, but no true optimization was done. Still, the topologies showed an average number of hops very close to that of the ideal spanning tree. The deviation from this ideal situation is shown in Figure 1. For small networks $(N<18)$, we have found topologies that give an $H$ very close to the ideal. The deviations for $N=24$ is likely to be due to limitations in the search for the optimum topology.

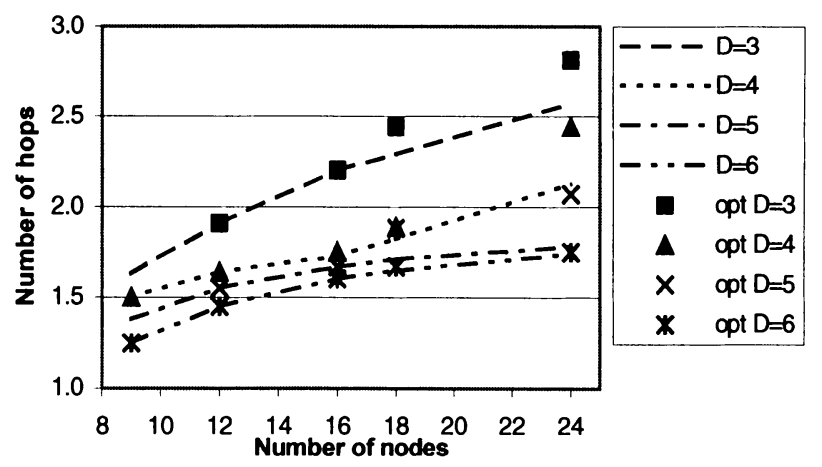

Figure 1: Comparison of the average number of hops for the 'optimized' topologies (markers) and for the ideal spanning tree (lines). 
The results of the average number of hops simulations are shown in Figure 2 and 3. The different topologies considered (see legends used in Figure 2 and 3 ) included:

- 'opt $D$ '

optimized to give a minimum average number of hops for a given node degree

- 'torus' torus-like topologies where we have connected the network boundaries

- 'square' regular grid topologies

- 'cylindrical' regular grid topology on a cylindrical surface

- 'double ring' two ring networks interconnected with 4 links

- 'triangular' two ring networks interconnected with 3 links

- 'diamond' regular grid limited to a diamond shaped area

For the first two topology categories all nodes have the same node degrees ('integer topologies'). The remaining topology categories have non-equal nodes and therefore non-integer average node degrees ('non-integer topologies').

From Figure 2 it follows that $H$ increases sub-linearly with the number of nodes for all topologies. The larger the node degree, the lower the rate of increase. For clarity we have not included the results for $D=2$, i.e. ring networks, where $H$ grows linearly with $N$. For a given network size, there is a significant reduction in $H$ in going from $D=2$ to 3 and 4. However, increasing $D$ to 5 and 6 results in only modest improvement (at least for the small to medium sized networks considered here). The optimized topologies reduce $H$ by approximately $20 \%(D=3,4)$ and $10 \%(D=6)$.

Our fourth conclusion is therefore that for a given node degree we can reduce $H$ significantly by optimizing the topology.

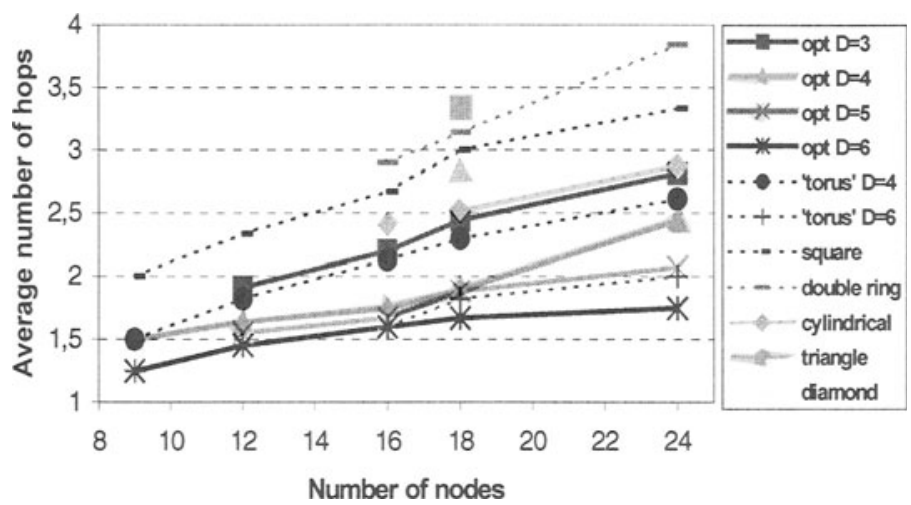

Figure 2: The average number of hops as function of number of nodes for different physical topologies. The various topologies are described in the text. 


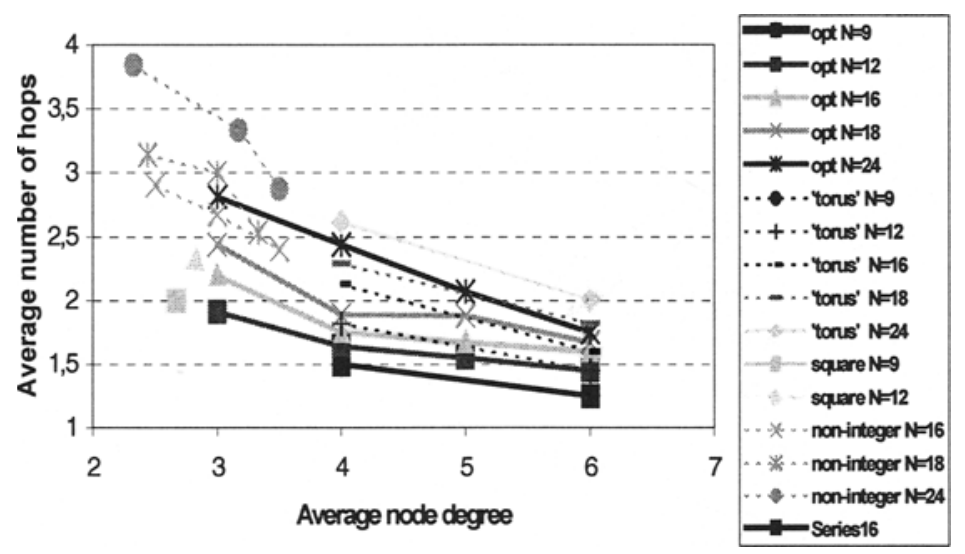

Figure 3: Average number of hops as function of average node degree for different physical topologies. For 'non-integer' topologies the data points are ordered such that the first point corresponds to 'double ring', the second to 'square', and the third to 'cylindrical'.

Figure 3 illustrates how $H$ varies with the average node degree for various network sizes. For all networks considered here, $H$ decrease with increasing node degree as expected. Again there is a large reduction in $H$ going from $D=3$ to 4 . The reduction is more modest for increasing $D$ from 4 to 6 . $H$ decreases by approximately $15-25 \%$ for $D$ increasing from 3 to 4 and from 4 to 6 . The relative decrease of $H$ is almost independent of network size and topology.

Our fifth conclusion is therefore that for a given network size $N$, the most effective way to reduce $H$ is to increase the node degree.

\section{MINIMUM OVERALL NETWORK COST TOPOLOGY}

The influence of physical topology on overall network cost is illustrated for an $N=12$ network in Figure 4. A ring, a regular grid, a torus, an optimized topology, and an optimized topology mapped onto a regular grid is compared. It follows that for this network size the best topology is a ring if cable cost dominates $(D L)$, a square or torus if fiber cost dominates $(H L)$, and an optimized topology if node cost dominates $(H)$. The best topology therefore depends on the relative importance of these three factors. 


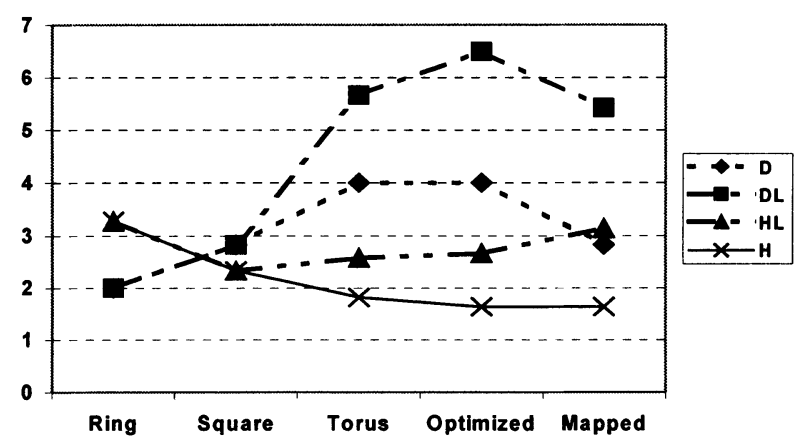

Figure 4: The three factors contributing to the network cost for an $\mathrm{N}=12$ network. We estimate the link length by placing the nodes on a grid and computing the Euclidean length of each network link.

Minimum- $H$ topologies are most effective at reducing the node complexity. They could therefore be attractive in situations where the main limitations are in the nodes. To simplify the network it is possible to map the minimum- $H$ topology onto a regular topology. The minimum- $H$ approach is then equivalent to regular topology approaches with fiber by-passes at the nodes. This will increase the fiber cost and reduce cable cost as shown in Figure 4.

\section{CONCLUSIONS}

We have shown how a small number of average network parameters can be used to guide the design and evaluation of WRONs. In particular, we have demonstrated that the average number of hops required to route the traffic demand is a key design parameter for such networks.

We have demonstrated that the overall network implementation cost is determined by the average number of hops required, $H$, the node degree, $D$, and the average link length, $L$. Using this, it is shown how the best topology (lowest cost) shifts from a sparsely connected topology if the cable cost dominates, to medium connected topology if the fiber cost is dominating, to densely connected topologies if the node cost is dominating.

The lowest $H$ (and lowest node complexity) are found for highly non-regular topologies requiring long link lengths. These lowest $H$ are found to be close to those of the case of an ideal spanning trees. It should be noted that the optimized topologies can be mapped onto regular grids by using fiber bypasses at the node locations. Compared to the ordinary regular grid networks, the optimized topologies will have reduced node complexities. This is achieved by properly sorting the traffic in the cable/fibers at the source node according to connections (destination node). 


\section{REFERENCES}

D.R. Hjelme et.al. (1996), 'How many wavelengths does it take to build a wavelength routed optical network?', ECOC’97, paper MOA.4.2, Oslo, Norway, 1996.

S. Baroni et. al. (1997), 'Wavelength requirements in arbitrarily connected wavelength-routed optical networks,' Journal of Ligthwave Technol., vol 15, pp 242-251, 1997.

D.R. Hjelme, (1997) 'OXC components count as function of traffic scaling,' ACTS OPEN meeting, Oslo, Norway, 1997, unpublished.

E. Limal, K.E. Stubkjær, and D.R. Hjelme (1998), 'Optimization of the transmission system for wavelength routed optical network: trade-off between node complexity and transmission channel utilization,' submitted to NOC'98, Manchester, UK, 1998

A. Jourdan et.al. (1996), 'Design and implementation of a fully reconfigurable all-optical crossconnect for high capacity multiwavelength transport networks,' Journal of Lightwave Technol., vol 14, pp1198-1206, 1996.

M.G. Hluchyj et. al. (1991), 'ShuffleNet: an application of generalized perfect shuffles to multihop lightwave networks,' Journal of Lightwave Technol., vol 9, pp 1386-1397, 1991.

\section{BIOGRAPHY}

Dag Roar Hjelme received his Diploma degree in Electrical Engineering from the Norwegian Institute of Technology in 1982 and his Ph.D. degree in Electrical Engineering from University of Colorado (USA) in 1988. From 1988 till 1992 he was a Post.Doc and staff researcher with the University of Colorado. Since 1993 he has been with SINTEF, Trondheim (Norway), where he is currently a Senior Scientist. In 1995 he was appointed Adjunct Professor of Optical Communications at the Norwegian University of Science and Technology.

Stig Eikedal Andersen received his Diploma degree in Electrical Engineering from the Department of Physical Electronics at the Norwegian University of Science and Technology, Trondheim, in 1997. 